\title{
Serum-free medium and hypoxic preconditioning synergistically enhance the therapeutic effects of mesenchymal stem cells on experimental renal fibrosis
}

Naoki Ishiuchi ${ }^{1,2,3}$, Ayumu Nakashima ${ }^{1,4^{*}} \mathbb{D}$, Shigehiro Doi ${ }^{1}$, Ryo Kanai ${ }^{1}$, Satoshi Maeda ${ }^{4,5}$, Shinya Takahashi ${ }^{6}$, Masataka Nagao ${ }^{3}$ and Takao Masaki ${ }^{{ }^{*}}$

\begin{abstract}
Background: Mesenchymal stem cells (MSCs) repair injured tissue in a paracrine manner. To enhance their therapeutic properties, preconditioning with various factors has been researched. We have previously showed that MSCS cultured in serum-free medium (SF-MSCs) promote their immunosuppressive ability, thereby enhancing their antifibrotic effect. Here, we examined whether serum-free medium and hypoxic preconditioning synergistically enhance the therapeutic effects of MSCs on renal fibrosis in rats with ischemia-reperfusion injury (IRI).

Methods: SF-MSCs were incubated under $1 \% \mathrm{O}_{2}$ conditions (hypo-SF-MSCs) or $21 \% \mathrm{O}_{2}$ conditions (normo-SF-MSCs) for $24 \mathrm{~h}$ before collection. After IRI procedure, hypo-SF-MSCs or normo-SF-MSCs were injected through the abdominal aorta. At 7 or 21 days post-injection, the rats were killed and their kidneys were collected to evaluate inflammation and fibrosis. In in vitro experiments, we investigated whether hypo-SF-MSCs enhanced secretion of anti-fibrotic humoral factors using transforming growth factor (TGF)- $\beta 1$-stimulated HK-2 cells incubated with conditioned medium from hypo-SF-MSCs or normo-SF-MSCS.

Results: Normo-SF-MSCs showed attenuation of senescence, which increased their proliferative capacity. Although no significant difference in cellular senescence was found between normo-SF-MSCs and hypo-SF-MSCs, hypo-SFMSCs further increased their proliferative capacity compared with normo-SF-MSCs. Additionally, administration of hypo-SF-MSCs more strongly ameliorated renal fibrosis than that of normo-SF-MSCs. Moreover, although hypo-SFMSCs strongly attenuated infiltration of inflammatory cells compared with the control rats, which were treated with PBS, this attenuation was almost equal between normo-SF-MSCs and hypo-SF-MSCs. In vitro experiments revealed that hypo-SF-MSCs more significantly inhibited transforming growth factor (TGF)- $\beta / S$ mad signaling compared with normo-SF-MSCs. Moreover, hypoxic preconditioning increased hepatocyte growth factor (HGF) secretion even under serum-free conditions, whereas knockdown of HGF in hypo-SF-MSCs attenuated inhibition of TGF- $\beta / S m a d$ signaling.
\end{abstract}

\footnotetext{
*Correspondence: ayumu@hiroshima-u.ac.jp; masakit@hiroshima-u.ac.jp

${ }^{1}$ Department of Nephrology, Hiroshima University Hospital, 1-2-3 Kasumi,

Minami-ku, Hiroshima, Hiroshima 734-8551, Japan

${ }^{4}$ Department of Stem Cell Biology and Medicine, Graduate School

of Biomedical \& Health Sciences, Hiroshima University, 1-2-3 Kasumi,

Minami-ku, Hiroshima, Hiroshima 734-8553, Japan

Full list of author information is available at the end of the article
}

(c) The Author(s) 2021. Open Access This article is licensed under a Creative Commons Attribution 4.0 International License, which permits use, sharing, adaptation, distribution and reproduction in any medium or format, as long as you give appropriate credit to the original author(s) and the source, provide a link to the Creative Commons licence, and indicate if changes were made. The images or other third party material in this article are included in the article's Creative Commons licence, unless indicated otherwise in a credit line to the material. If material is not included in the article's Creative Commons licence and your intended use is not permitted by statutory regulation or exceeds the permitted use, you will need to obtain permission directly from the copyright holder. To view a copy of this licence, visit http://creativecommons.org/licenses/by/4.0/. The Creative Commons Public Domain Dedication waiver (http://creativeco mmons.org/publicdomain/zero/1.0/) applies to the data made available in this article, unless otherwise stated in a credit line to the data. 
Conclusions: These results indicate that administration of ex vivo-expanded, hypoxia-preconditioned SF-MSCs may be a useful cell therapy to prevent renal fibrosis.

Keywords: Mesenchymal stem cells, Hypoxic preconditioning, Serum-free conditions, Hepatocyte growth factor, Renal fibrosis

\section{Background}

Acute kidney injury (AKI) is common clinical problem with high mortality and morbidity [1, 2], which is often caused by loss of blood flow to the kidney during volume depletion, shock, sepsis, and renal transplantation [3, 4]. In general, most AKI patients recover their baseline renal functions, but several studies have demonstrated that a large number of AKI patients eventually develop chronic kidney disease (CKD), which suggests that AKI, even if followed by renal recovery, is a major risk factor for progression of CKD $[5,6]$. Although the mechanism of AKI-to-CKD transition is incompletely understood, recent studies have reported that several pathological mechanisms, including hypoxia, microvascular rarefaction, inflammation, transforming growth factor (TGF)- $\beta 1$ production, and epithelial-mesenchymal transition, are involved in AKI-to-CKD transition [7, 8]. However, there are currently few effective therapies to prevent AKI-toCKD progression.

Mesenchymal stem cells (MSCs) are multipotent adult stem cells, which can be isolated from various tissues, such as bone marrow, adipose tissue, and umbilical cord $[9,10]$, and repair injured tissue via their paracrine effects $[11,12]$. Several studies have demonstrated that administration of MSCs exerts beneficial effects against various renal disease models $[13,14]$. However, the MSCs in these studies were cultured by the conventional method and did not exhibit sufficient therapeutic efficacy.

MSCs remain dormant under normal conditions, whereas MSCs activated by cytokines released from immune cells in damaged tissues are converted to the active form and exert anti-inflammatory effects $[15,16]$. This conversion to the active form might require several days. Therefore, prior to administration, preconditioning with various factors, including cytokines [17, 18], hypoxia [19, 20], pharmacological compounds [21, 22], and genetic modification [23, 24], has been widely studied to enhance the therapeutic effects of MSCs. We have previously reported that MSCs cultured in STK (Kanto Chemical, Tokyo, Japan), which is serum-free medium containing growth factors for MSC culture, promote their immunosuppressive ability by increasing the expression of tumor necrosis factor- $\alpha$-induced protein 6 (TSG6) and inducing polarization of immunosuppressive M2 macrophages, thereby enhancing their anti-fibrotic effect [25]. Furthermore, we have recently demonstrated that hypoxia-preconditioned MSCs have enhanced immunosuppressive and anti-fibrotic abilities by increased vascular endothelial growth factor (VEGF) and hepatocyte growth factor (HGF) secretion [20]. These findings led us to the hypothesis that serum-free conditions and hypoxic preconditioning would synergistically enhance the therapeutic effects of MSCs on renal fibrosis.

In this study using human bone marrow-derived MSCs, we show that MSCs cultured in serum-free medium under $1 \% \mathrm{O}_{2}$ conditions (hypo-SF-MSCs) potently ameliorate renal fibrosis in a rat ischemia-reperfusion injury (IRI) model. We also show that conditioned medium from hypo-SF-MSCs strongly inhibits TGF- $\beta 1$-induced fibrotic changes in HK-2 cells. Additionally, we demonstrate that inhibition of HGF reduces the anti-fibrotic effect of hypo-SF-MSCs. Our data suggest that administration of hypoxia-preconditioned SF-MSCs may be a useful therapy to prevent progression of renal fibrosis.

\section{Methods \\ Preparation of MSCs}

Human MSCs were isolated from bone marrow collected from the sternum of two patients who underwent coronary artery bypass grafting. The cells were cultured in Dulbecco's modified Eagle's medium (DMEM; SigmaAldrich, St. Louis, MO, USA) with 10\% FBS (SigmaAldrich) or STK and were designated as "10\%MSCs" or "SF-MSCs", respectively. Cells up to passage 6 were used in all experiments and counted by a TC-20 (Bio-Rad, Hercules, CA, USA). The Medical Ethics Committee of Hiroshima Graduate School of Biomedical Science permitted collection of the bone marrow (Permit number: E-1089, registered on February 2, 2018). Each patient provided written informed consent.

\section{Flow cytometric analysis}

Flow cytometric analysis was performed in accordance with previously described methods [25]. The following antibodies were used: anti-human CD29 IgG antibody (BioLegend, San Diego, CA), anti-human CD44 IgG antibody (BioLegend), anti-human CD73 IgG antibody (BioLegend), anti-human CD90 IgG antibody (BioLegend), anti-human CD11b IgG antibody (BioLegend), anti-human CD34 IgG antibody (BioLegend), antihuman CD45 IgG antibody (BioLegend), anti-human HLA-A,B,C IgG antibody (BioLegend), and anti-human 
HLA-DR IgG antibody (BioLegend). The stained MSCs were analyzed by a BD FACSVerse (Becton, Dickinson and Company, Franklin Lakes, NJ). Data were assessed by FlowJo software (FlowJo, LLC; Ashland, OR).

\section{Differentiation experiments}

To induce adipogenic or osteogenic differentiation, MSCs were cultured in adipogenic differentiation medium (Takara Bio, Shiga, Japan) or osteogenic differentiation medium (Sigma-Aldrich), respectively, for 14 days following the manufacturers' protocols. Oil Red O (SigmaAldrich) and Alizarin Red S (FUJIFILM Wako Pure Chemical, Osaka, Japan) were used to analyze adipogenic and osteogenic differentiation, respectively.

\section{Cell proliferation assay}

Proliferative activity of MSCs was analyzed by a watersoluble tetrazolium salt (WST)-1 assay (Takara Bio). MSCs $\left(2.5 \times 10^{3}\right.$ cells $\left./ 100 \mu \mathrm{L}\right)$ were seeded in 96-well microplates and cultured in DMEM containing 10\% FBS or STK under normoxic $\left(21 \% \mathrm{O}_{2}\right)$ or hypoxic $(1 \%$ $\mathrm{O}_{2}$ ) conditions. After incubation for 0,12 , and $24 \mathrm{~h}, 10$ $\mu \mathrm{L}$ WST-1 reagent was added to each well, followed by incubation for $4 \mathrm{~h}$. Absorbance was determined using a microplate reader at a wavelength of $450 \mathrm{~nm}$ and reference wavelength of $620 \mathrm{~nm}$.

\section{Transwell migration assay}

The migration capacity of MSCs was analyzed using a Cell Migration/Chemotaxis Assay Kit (PromoCell, Heidelberg, Germany) containing transwell chambers (24well, $8 \mu \mathrm{m}$ pore size). $10 \%$ MSCs and SF-MSCs $\left(2 \times 10^{5}\right.$ cells/well) were seeded in upper chamber and $600 \mu \mathrm{l}$ serum-free medium containing stromal cell-derived factor 1 (100 ng/ml, PEPROTECH, Cranbury, NJ, USA) was placed in the lower chamber. After $10 \mathrm{~h}$ of incubation under normoxic $\left(21 \% \mathrm{O}_{2}\right)$ or hypoxic $\left(1 \% \mathrm{O}_{2}\right)$ conditions, cells on the upper side of the membrane were removed with a cotton swab. Then, the upper chamber was removed and medium in the lower chamber was replaced with $550 \mu \mathrm{l}$ of cell dissociation solution containing a dye solution. Subsequently, the upper chamber was placed in the lower chamber, followed by $1 \mathrm{~h}$ of incubation under normoxia $\left(21 \% \mathrm{O}_{2}\right)$ and then $110 \mu \mathrm{l}$ of the mixture in the lower chamber was transferred to a 96-well plate. A standard curve of cells that contained a dye solution was also generated in the 96-well plate following the manufacturer's protocol. This plate was read using the fluorescence plate reader at 530/590 nm (Ex/Em). The number of migrated cells was calculated using the standard curve.

\section{Hypoxic preconditioning}

For hypoxic preconditioning, MSCs were cultured in STK. At 70\% confluence, the culture medium was replaced with fresh complete medium and the cells were incubated under $1 \% \mathrm{O}_{2}$ conditions (hypo-SF-MSCs) or $21 \% \mathrm{O}_{2}$ conditions (normo-SF-MSCs) for $24 \mathrm{~h}$ before collection. Hypoxic preconditioning was performed in a Modular Incubator Chamber (MIC 101) (Billups-Rothenberg, San Diego, CA, USA). 10\%MSCs were only incubated under $21 \% \mathrm{O}_{2}$ conditions (normo-10\%MSCs).

\section{Animals}

Male Sprague-Dawley (SD) rats (8 weeks old) were purchased from Charles River Laboratories Japan (Yokohama, Japan) and were used to induce IRI. All experimental procedures were approved by the Institutional Animal Care and Use Committee of Hiroshima University (Hiroshima, Japan) (Permit number: A1683) and conducted in accordance with the "Guide for the Care and Use of Laboratory Animals, $8^{\text {th }}$ ed, 2010" (National Institutes of Health, Bethesda, MD, USA).

\section{Experimental animal model}

$\mathrm{SD}$ rats were randomly divided into four groups $(n=5$ in each group): sham, PBS, normo-SF-MSCs, and hypoSF-MSCs groups. Renal IRI was induced by transiently clamping the unilateral renal artery. Rats were anesthetized by an intraperitoneal injection of three types of mixed anesthetic agents (medetomidine, midazolam, and butorphanol). After performing a laparotomy, the left kidney was exposed. Then, the renal pedicle was clamped using atraumatic vascular clamps for $1 \mathrm{~h}$, followed by reperfusion on a heating blanket. After reperfusion, MSCs $\left(5 \times 10^{5}\right.$ cells $\left./ \mathrm{rat}\right)$ in $0.2 \mathrm{ml}$ PBS were injected into the abdominal aorta clamped above and below the left renal artery bifurcation. At 7 or 21 days post-injection, the rats were killed and their left kidneys were collected to evaluate inflammation and fibrosis.

\section{Western blot analysis}

Sample collection and western blotting were performed as described previously [20]. Rabbit polyclonal antiSirt1 antibody (Sigma-Aldrich), rabbit monoclonal antip16 ${ }^{\text {INK4a }}$ antibody (Abcam, Cambridge, UK), mouse monoclonal anti-GAPDH antibody (Sigma-Aldrich), mouse monoclonal anti- $\alpha$-SMA antibody (SigmaAldrich), mouse monoclonal anti-TGF- $\beta 1$ antibody (Santa Cruz Biotechnology, Santa Cruz, CA, USA), rabbit monoclonal anti-phosphorylated Smad2 (p-Smad2) antibody (Cell Signaling Technology, Danvers, MA, USA), mouse monoclonal anti-Smad2 antibody (Cell Signaling Technology), mouse monoclonal anti- $\alpha$-tubulin antibody 
(Sigma-Aldrich), rabbit monoclonal anti-CD163 antibody (Abcam), and rabbit polyclonal anti-CD68 antibody (Abcam) were used as primary antibodies. Horseradish peroxidase-conjugated goat anti-rabbit immunoglobulin G (Dako, Glostrup, Denmark) or goat anti-mouse immunoglobulin G (Dako) were used as secondary antibodies. SuperSignal West Dura or Pico system (Thermo Fisher Scientific, Rockford, IL, USA) was used to detect signals. The intensity of each band was analyzed by ImageJ software (version 1.47v; National Institutes of Health) and standardized by the level of either GAPDH or $\alpha$-tubulin.

\section{Immunohistochemical analysis}

Immunohistochemical staining of formalin-fixed, paraffin-embedded tissue Sects. ( $4 \mu \mathrm{m}$ thick) was performed in accordance with previously described methods [25]. The following primary antibodies were used: mouse monoclonal anti- $\alpha$-SMA antibody (Sigma-Aldrich), rabbit polyclonal anti-CD3 antibody (Dako), rabbit polyclonal anti-CD68 antibody (Abcam), and rabbit monoclonal anti-CD163 antibody (Abcam). CD3-, CD68-, and CD163-positive cells and positive areas for $\alpha$-SMA staining were determined using Image software by examination of five randomly selected fields $(\times 200)$ of the cortex.

\section{Histological analysis}

Formalin-fixed, paraffin-embedded tissue Sects. $(2 \mu \mathrm{m}$ thick) were stained with hematoxylin and eosin (HE) and Masson trichrome to assess histological injury and fibrosis. The areas of interstitial fibrosis were evaluated using Lumina Vision (Mitani, Osaka, Japan) by examining five randomly selected fields $(\times 200)$ of the cortex.

\section{Preparation of conditioned medium}

To generate conditioned medium from normo-SF-MSCs (normo-SF-MSC-CM) and hypo-SF-MSCs (hypo-SFMSC-CM), MSCs $\left(3 \times 10^{5}\right.$ cells/dish) were seeded in $10-\mathrm{cm}$ dishes and cultured in DMEM containing $10 \%$ FBS or STK. At $70 \%$ confluence, the culture medium was replaced with DMEM containing $0.1 \% \mathrm{FBS}$, and the cells were cultured for 24 or $48 \mathrm{~h}$ under $1 \% \mathrm{O}_{2}$ or $21 \% \mathrm{O}_{2}$ conditions. Then, each medium was collected.

\section{Cell culture and treatments}

HK-2 cells were purchased from the American Type Culture Collection (Manassas, VA, USA). The cells were cultured as described previously [25]. After serum starvation of HK-2 cells with DMEM containing 0.1\% FBS or conditioned medium from MSCs for $24 \mathrm{~h}, 10 \mathrm{ng} / \mathrm{ml}$ recombinant human TGF- $\beta 1$ (R\&D Systems, Minneapolis, MN, USA) was added to the cells directly. After $30 \mathrm{~min}$ (to investigate protein levels of $\mathrm{p}-\mathrm{Smad} 2$ ) or $24 \mathrm{~h}$ (to investigate protein levels of $\alpha$-SMA), HK-2 cells were collected and subjected to in vitro experiments.

THP-1 cells were also obtained from the American Type Culture Collection and cultured as described previously [25]. To induce differentiation of THP-1 cells into M1 macrophages, THP-1 cells were treated with $160 \mathrm{nM}$ phorbol 12-myristate 13-acetate (Sigma-Aldrich) for $48 \mathrm{~h}$. Then, the medium was replaced with conditioned medium from MSCs. After $24 \mathrm{~h}$, the cells were collected and subjected to in vitro experiments.

\section{Quantitative real-time reverse transcription-PCR}

RNA extraction and real-time reverse transcriptionPCR were performed in accordance with previously described methods [25]. Specific oligonucleotide primers and probes for rat TGF- $\beta 1$ (assay ID: Rn00572010_m1), rat collagen type I (assay ID: Rn01463848_m1), rat collagen type III (assay ID: Rn01437681_m1), rat interleukin (IL)-1 $\beta$ (assay ID: Rn00580432_m1), rat IL-6 (assay ID: Rn01410330_m1), human TSG-6 (assay ID: Hs00200180_ $\mathrm{m} 1$ ), and 18S rRNA (endogenous control) were obtained as TaqMan Gene Expression Assays (Applied Biosystems, Foster City, CA, USA). mRNA levels were normalized to the level of 18S rRNA.

\section{Enzyme-linked immunosorbent assay (ELISA)}

ELISA analyses of VEGF (R\&D Systems) and HGF (R\&D Systems) were performed following the manufacturer's protocols. Concentrations were normalized to the total protein content.

\section{Transfection of HGF siRNA}

MSCs were transfected with $20 \mathrm{nM}$ siRNA that targeted HGF (s6530; Applied Biosystems) or negative control siRNA (4,390,843; Applied Biosystems) using Lipofectamine 2000 Transfection Reagent (Thermo Fisher Scientific). After $24 \mathrm{~h}$, the transfected cells were washed and fresh complete medium was added. At $80 \%$ confluence, these cells were used to generate conditioned medium.

\section{Statistical analysis}

Results are expressed as means \pm standard deviations (S.D.). For multiple group comparisons, one-way ANOVA followed by Bonferroni's post-hoc test was applied. Comparisons between two groups were analyzed by the Student's t-test. $P<0.05$ was considered statistically significant.

\section{Results \\ Characterization of MSCs}

We first identified MSCs by morphology and flow cytometry. The cells displayed typical spindle shapes and were 
adherent to plastic dishes. Flow cytometry showed that the cells expressed standard MSC markers, such as CD29, CD44, CD73, CD90, and HLA-A,B,C (Additional file 1a), and did not express MSC-negative markers, such as CD11b, CD34, CD45, and HLA-DR (Additional file 1a). Additionally, we assessed the adipogenic and osteogenic differentiation abilities of MSCs. Oil Red O and Alizarin Red S staining showed that the MSCs could undergo adipogenic and osteogenic differentiations (Additional file 1b, 1c).

\section{Serum-free medium ameliorates senescence of MSCs}

In accordance with previous studies, MSCs cultured for many passages exhibit a senescence-like morphology such as irregular shapes, enlarged cell bodies, and flattened cell bodies [26, 27]. However, unlike 10\%MSCs, SF-MSCs at passage 8 did not display senescence-like morphology, but elongated spindle shapes, whereas their morphology was almost equivalent in SF-MSCs cultured under normoxia and hypoxia (Fig. 1a). Several studies have reported that downregulated expression of Sirt1 is involved in the aging process of MSCs with evidence that senescence-associated factor $\mathrm{p} 16^{\mathrm{INK} 4 \mathrm{a}}$ is negatively regulated by Sirt1 $[28,29]$. Accordingly, we next examined the expression of Sirt1 and p16 $6^{\text {INK4a }}$ in MSCs. Although the protein level of Sirt1 was upregulated in SF-MSCs compared with normo-10\%MSCs, there was no significant difference between normo-SF-MSCs and hypo-SFMSCs (Fig. 1b). Conversely, expression of p16 ${ }^{\mathrm{INK} 4 \mathrm{a}}$ was decreased in both normo-SF-MSCs and hypo-SF-MSCs compared with normo-10\%MSCs, but there was no significant difference between normo-SF-MSCs and hypoSF-MSCs (Fig. 1b).

\section{Hypoxic preconditioning enhances the proliferative ability and migration capacity of SF-MSCs}

To assess whether the combination of serum-free medium and hypoxic preconditioning promoted the proliferative ability of MSCs, we examined the proliferative activity of normo-10\%MSCs, normo-SF-MSCs, and hypo-SF-MSCs using the WST-1 assay. The absorbance value of normo-SF-MSCs was more significantly increased than that of normo- $10 \% \mathrm{MSCs}$ in a timedependent manner and a further increase was observed in hypo-SF-MSCs (Fig. 1c), which suggested that serum-free medium and hypoxic preconditioning synergistically enhanced the proliferative ability of MSCs. Additionally, we investigated whether the combination of serum-free conditions and hypoxic preconditioning enhanced the migration capacity of MSCs by the transwell migration assay. As shown in Fig. 1d, the migration ability of normo-SF-MSCs was lower than that of normo-10\%MSCs, but this reduction was canceled in hypo-SF-MSCs.

\section{Hypoxia-preconditioned SF-MSCs attenuate IRI-induced renal fibrosis in rats}

To clarify the effect of hypoxia-preconditioned SFMSCs on renal fibrosis, we examined the expression of $\alpha$-SMA and TGF- $\beta 1$ in the IRI model that had been injected with PBS, normo-SF-MSCs, or hypo-SF-MSCs at 21 days post-IRI. As shown in Fig. 2a, the protein level of $\alpha$-SMA was remarkably increased in IRI rats injected with PBS (PBS group). This increase was suppressed in IRI rats injected with normo-SF-MSCs (normo-SF-MSCs group) and further suppression was observed in those injected with hypo-SF-MSCs (hypoSF-MSCs group) (Fig. 2a). In contrast, upregulation of the protein and mRNA levels of TGF- $\beta 1$ in the IRI model was reduced by MSC treatments with no significant difference between normo-SF-MSCs and hypoSF-MSCs groups (Fig. 2b, c). However, collagen type I and III mRNA levels were more reduced in the hypoSF-MSCs group than in the normo-SF-MSCs group (Fig. 2c). Moreover, HE staining showed tubular dilatation and tubular cast formation in the PBS group and these tubulointerstitial injuries were suppressed by MSC treatments, particularly in the hypo-SF-MSCs group (Fig. 2d). Masson trichrome staining revealed that the area of interstitial fibrosis was more significantly reduced in the hypo-SF-MSCs group than in the normo-SF-MSCs group (Fig. 2d, e). Similarly, immunostaining revealed that the $\alpha$-SMA-positive area was reduced in the hypo-SF-MSCs group compared with the normo-SF-MSCs group (Fig. 2d, e).

\footnotetext{
(See figure on next page.)

Fig. 1 Hypo-SF-MSCs have ameliorated senescence and an enhanced proliferative ability. a Representative images of normo-10\%MSCs, normo-SF-MSCs, and hypo-SF-MSCs at passage 8 (scale bar $=100 \mu \mathrm{m})$. b Western blot analysis of Sirt1 and p16 ${ }^{\text {INK4a }}$ in 10\%MSCs, normo-SF-MSCS, and hypo-SF-MSCS. Graphs show densitometric analyses of Sirt 1 and p1 $6^{\text {INK4a }}$ expression levels normalized to the GAPDH expression level $(n=5$ in each group). c Surviving cells were assessed by a WST-1 assay. Graph shows the absorbance value at each time point $(0,12$, and $24 \mathrm{~h})(n=5$ in each group). ${ }^{\dagger} P<0.01$ versus normo-10\%MSCs, ${ }^{\ddagger} P<0.01$ versus normo-SF-MSCs. $\mathbf{d}$ Migration activity of MSCs was analyzed using a transwell migration assay. Graph shows the numbers of migrated cells at $10 \mathrm{~h}(n=4$ in each group). Experiments to evaluate cell proliferation and migration were performed three times and similar results were obtained. Data are means \pm S.D. ${ }^{\#} P<0.01$ (one-way ANOVA followed by Bonferroni's post-hoc test)
} 
a

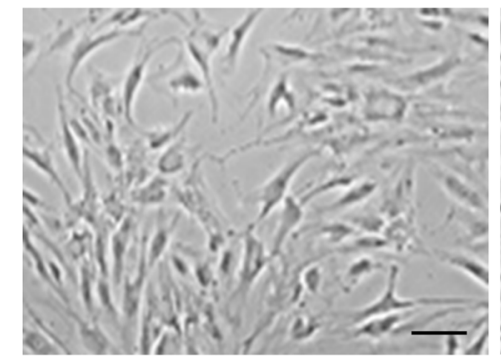

normo-10\%MSCs

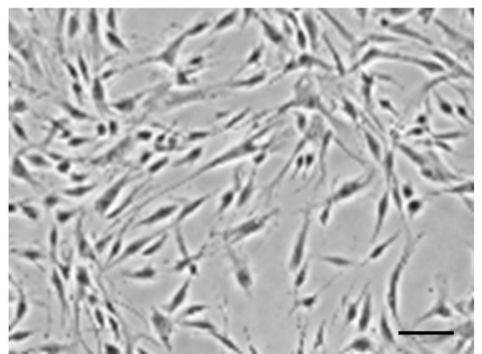

normo-SF-MSCs

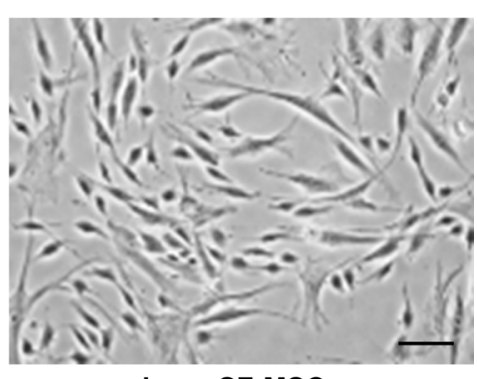

hypo-SF-MSCs

b
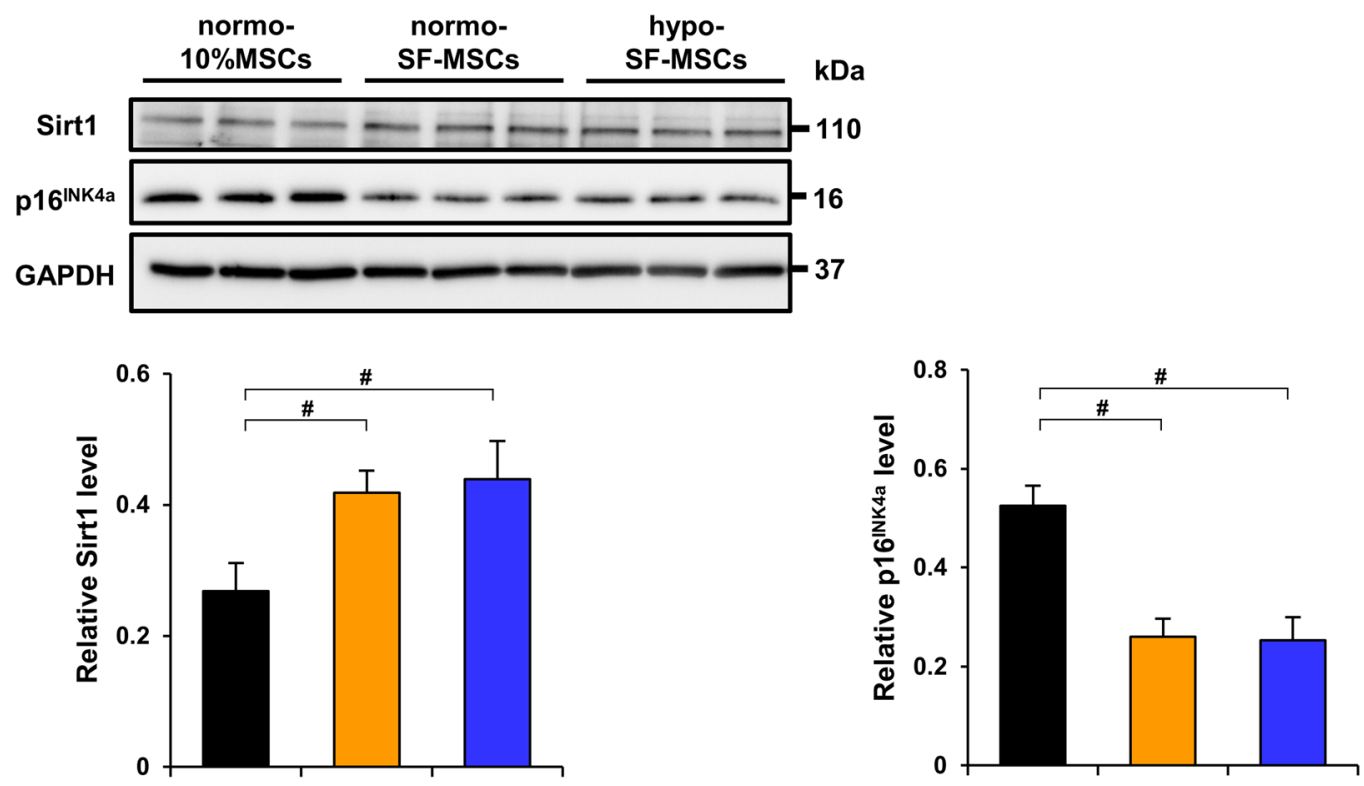

$\square_{10 \% \mathrm{MSC}}^{\text {normo- }}$

$\square$ normo-

$\square \begin{aligned} & \text { hypo- } \\ & \text { SF-MSCs }\end{aligned}$

C

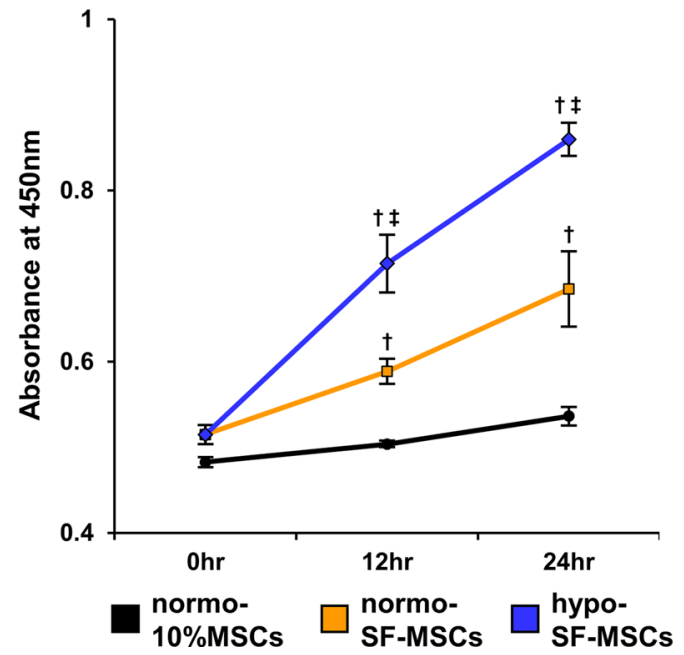

d

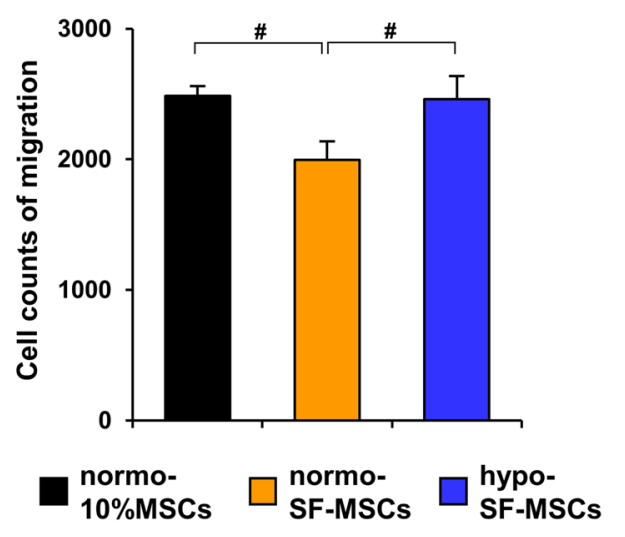

Fig. 1 (See legend on previous page.) 


\section{Hypoxia-preconditioned SF-MSCs suppress infiltration of inflammatory cells in IRI rats}

Several studies have reported that persistence of inflammation leads to progression of fibrosis [30, 31]. Therefore, we next analyzed the expression of CD3 (T cell marker), CD68 (M1 and M2 macrophage marker), and CD163 (M2 macrophage marker) at 7 days post-IRI to investigate the anti-inflammatory effect of hypoxia-preconditioned SFMSCs. As shown in Fig. 3a and b, immunohistochemical staining revealed that the number of CD3- and CD68positive cells was increased in the PBS group. The infiltration of these cells was inhibited by MSC treatments with no significant difference between normo-SF-MSCs and hypo-SF-MSCs groups (Fig. 3a, b). Conversely, the number of cells positive for CD163, an immunosuppressive macrophage marker, was upregulated in both SF-MSCs groups with no significant difference between normoSF-MSCs and hypo-SF-MSCs groups (Fig. 3a, b). We also found that IL-1 $\beta$ and IL- 6 mRNA levels were upregulated in the PBS group, and these increases were suppressed in both normo-SF-MSCs and hypo-SF-MSCs groups with no significant difference between them (Fig. 3c).

\section{Hypoxic preconditioning does not affect the SF-MSC-induced change in the phenotype of macrophages from $M 1$ to $M 2$}

We have previously demonstrated that SF-MSCs change the phenotype of macrophages from proinflammatory M1 to immunosuppressive M2 compared with $10 \%$ MSCs [25]. Therefore, we investigated whether hypoSF-MSCs further induced this change compared with normo-SF-MSCs. Although the protein level of CD163 was increased by both treatments with SF-MSC-CM, there was no significant difference between normo-SFMSC-CM and hypo-SF-MSC-CM (Fig. 4a). Additionally, we found that the TSG-6 mRNA level in SF-MSCs was higher than that in normo-10\%MSCs, but no significant difference was observed between normoxic and hypoxic conditions (Fig. 4b).

\section{Conditioned medium from hypoxia-preconditioned SF-MSCs strongly suppresses fibrotic changes by inhibition of TGF- $\beta /$ Smad signaling}

We found that hypoxic preconditioning did not promote the anti-inflammatory effect of SF-MSCs despite the fact that it enhanced the anti-fibrotic effect. Therefore, we investigated whether hypo-SF-MSCs enhanced inhibition of TGF- $\beta /$ Smad signaling using conditioned medium. To elucidate the direct anti-fibrotic effect of hypo-SF-MSCs on TGF- $\beta /$ Smad signaling, we measured the protein levels of $\mathrm{p}$-Smad2 and $\alpha$-SMA in TGF- $\beta 1$-stimulated HK-2 cells. We prepared normo-SF-MSC-CM and hypo-SFMSC-CM and then stimulated HK-2 cells with TGF- $\beta 1$ with or without each conditioned medium. The protein level of $\mathrm{p}-\mathrm{Smad} 2$ was upregulated by TGF- $\beta 1$ stimulation. This upregulation was reduced by treatment with normo-SF-MSC-CM and a further reduction was found after treatment with hypo-SF-MSC-CM (Fig. 5a). Similar results were observed for $\alpha$-SMA protein expression (Fig. 5b).

\section{Hypoxic preconditioning enhances VEGF and HGF secretion from SF-MSCs}

We previously found that hypoxic preconditioning has increased secretion of several renoprotective humoral factors, including VEGF and HGF [20]. To investigate whether hypoxic preconditioning enhanced secretion of these factors even under serum-free conditions, we measured the concentrations of VEGF and HGF in hypoSF-MSC-CM and normo-SF-MSC-CM by ELISAs. As shown in Fig. 6a and b, we found upregulation of VEGF and HGF in hypo-SF-MSC-CM compared with normoSF-MSC-CM. Next, to investigate the relationship between the duration of exposure to hypoxic conditions and VEGF secretion, SF-MSCs were incubated under $1 \%$ $\mathrm{O}_{2}$ conditions for 6,12 , or $24 \mathrm{~h}$. ELISA analysis showed that the concentration of VEGF was increased over time for $24 \mathrm{~h}$ (Fig. 6c). Additionally, we cultured SF-MSCs with various $\mathrm{O}_{2}$ concentrations for $24 \mathrm{~h}$. As shown in Fig. $6 \mathrm{~d}$, the concentration of VEGF was increased as the $\mathrm{O}_{2}$ concentration was decreased.

\section{Knockdown of HGF in hypoxia-preconditioned SF-MSCs reduces the anti-fibrotic effect in TGF- $\beta 1$-stimulated HK-2 cells \\ We found that the HGF concentration in hypo-SF- MSC-CM was higher than that in normo-SF-MSC-CM. Hence, to identify the effect of HGF secreted from MSCs on TGF- $\beta /$ Smad signaling, we prepared conditioned medium from hypo-SF-MSCs transfected with HGF}

\footnotetext{
(See figure on next page.)

Fig. 2 Hypo-SF-MSCs attenuate renal fibrosis more strongly than normo-SF-MSCs in IRI rats. a, b Western blot analysis of a-SMA and TGF- $\beta 1$ in the kidney cortex of IRI rats at day 21 post-IRI. Graphs show densitometric analyses of a-SMA and TGF- $\beta 1$ expression levels normalized to the GAPDH expression level ( $n=5$ in each group). c Expression levels of TGF- $\beta 1$ and collagen type I and III mRNAs in the kidney cortex of IRI rats at day 21 post-IRI ( $n=5$ in each group). $\mathbf{d}$ Representative images of immunohistochemical staining of a-SMA as well as HE and Masson trichrome staining of kidney sections at day 21 post-IRI (scale bar $=100 \mu \mathrm{m}$ ). e Quantification of the interstitial fibrosis area and a-SMA-positive area as percentages of the total area ( $n=5$ in each group). Data are means \pm S.D. ${ }^{*} P<0.01,{ }^{*} P<0.05$ (one-way ANOVA followed by Bonferroni's post-hoc test)
} 

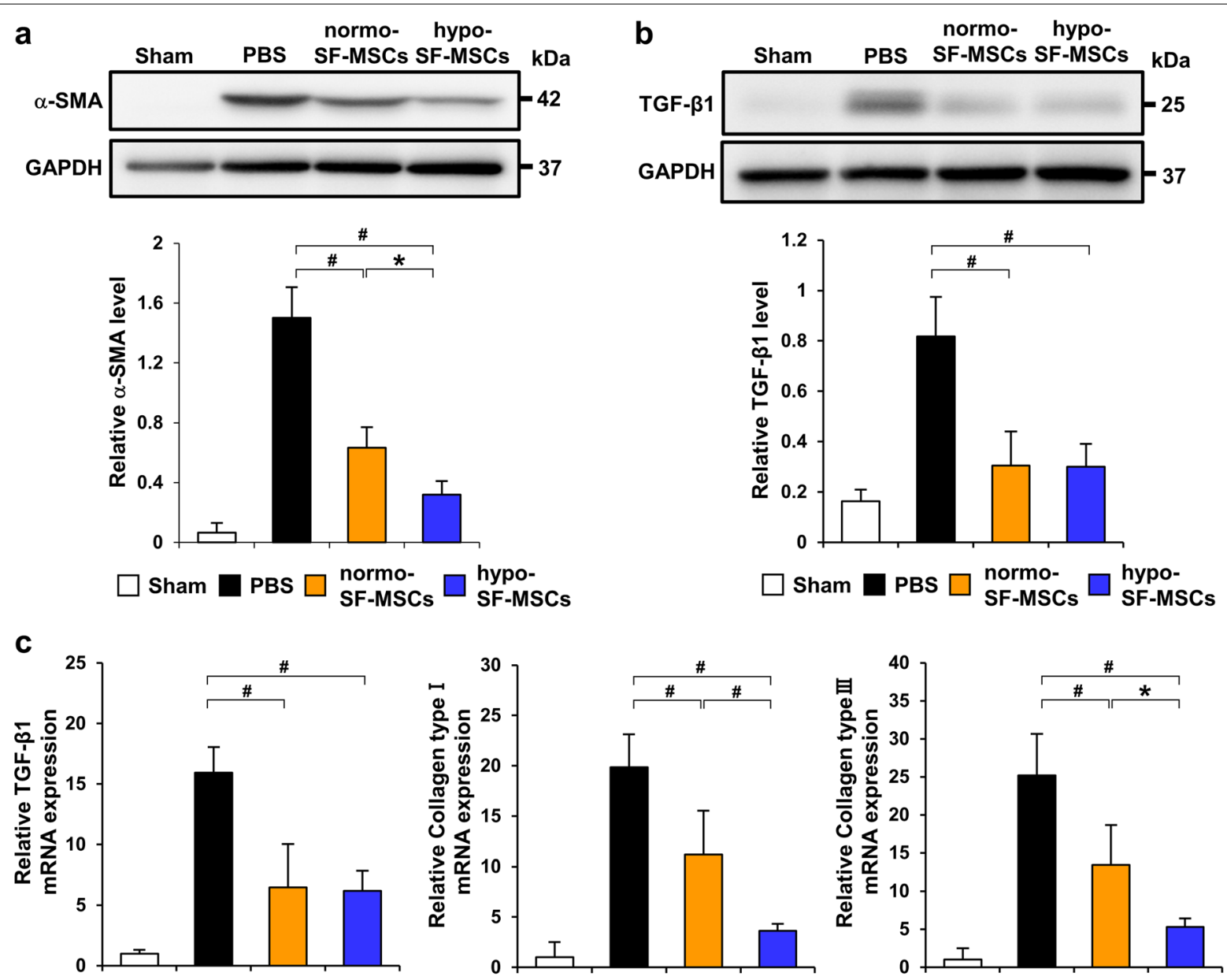

d
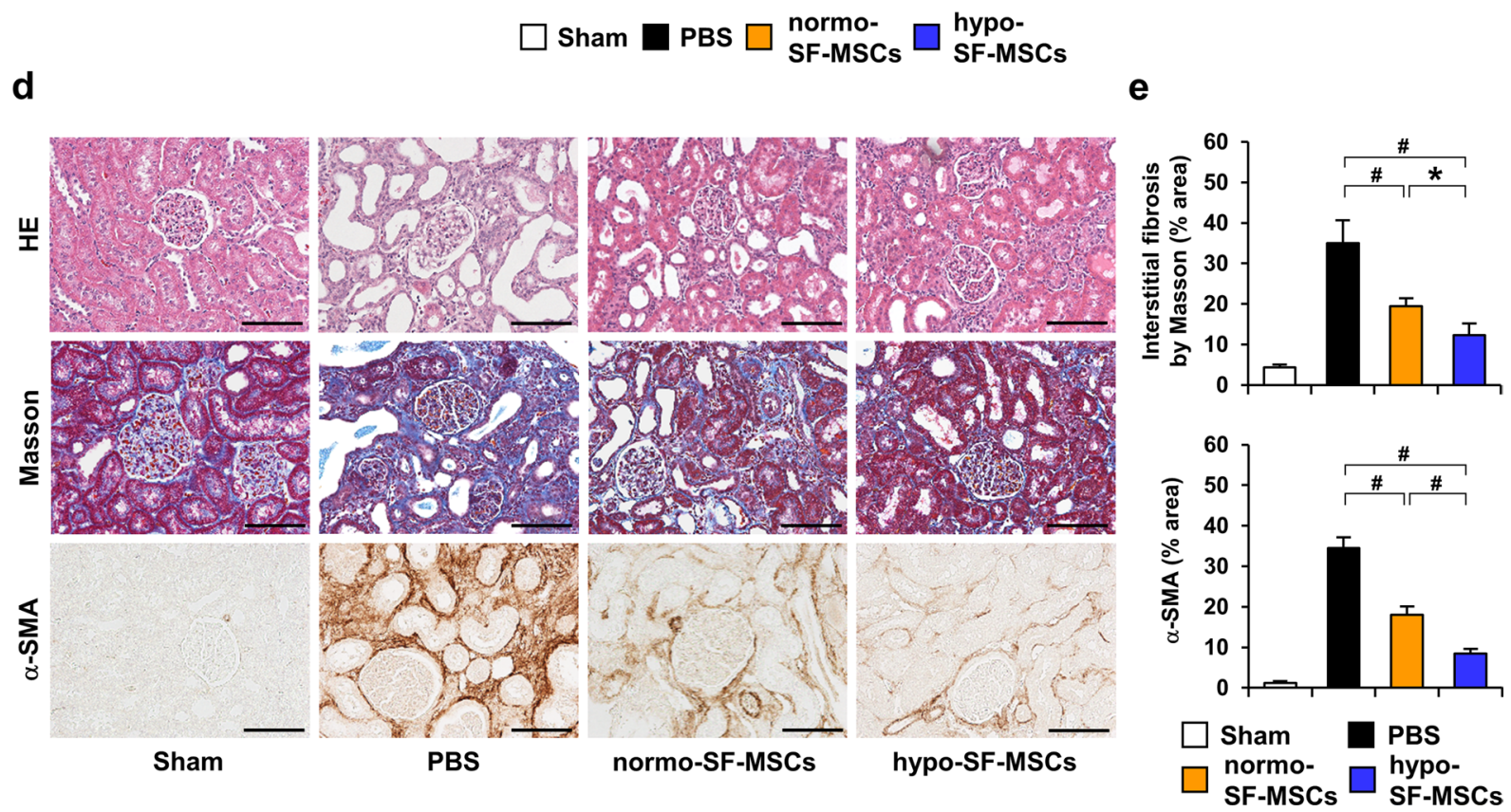

Fig. 2 (See legend on previous page.) 


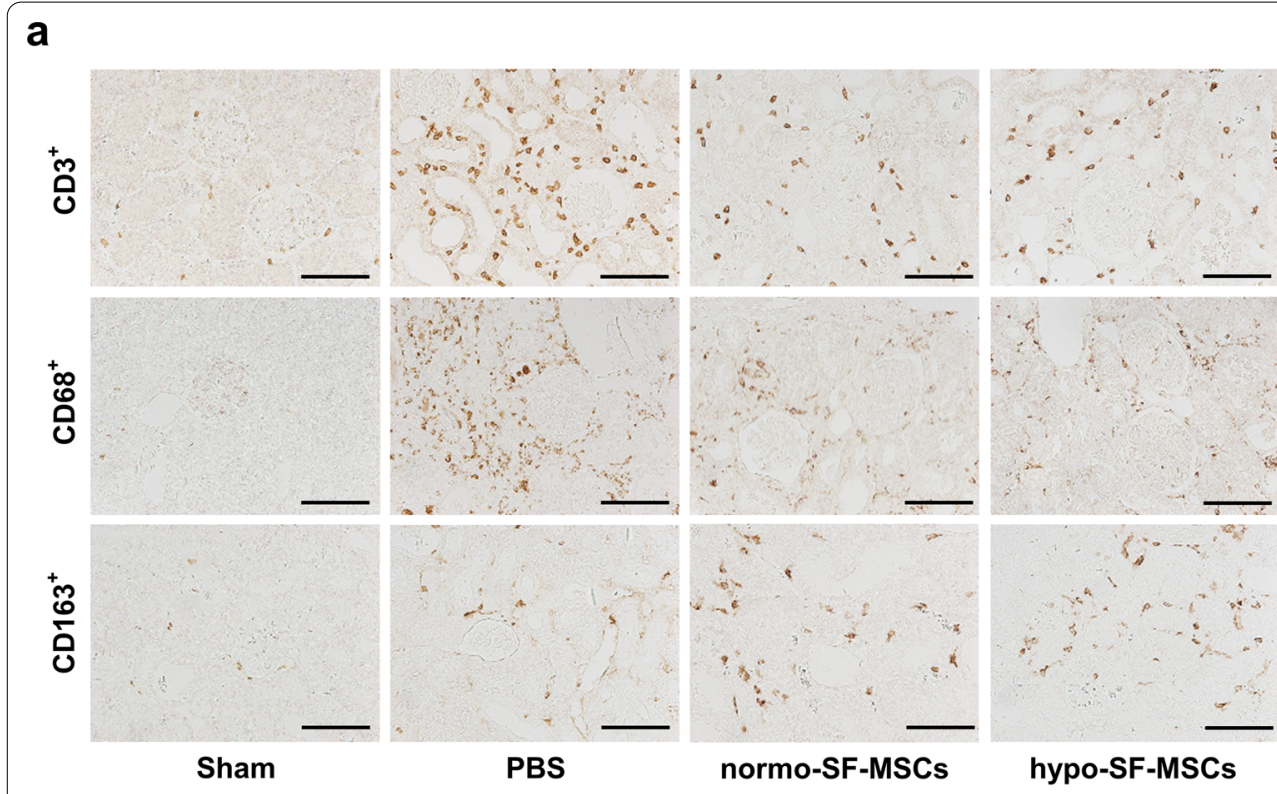

b
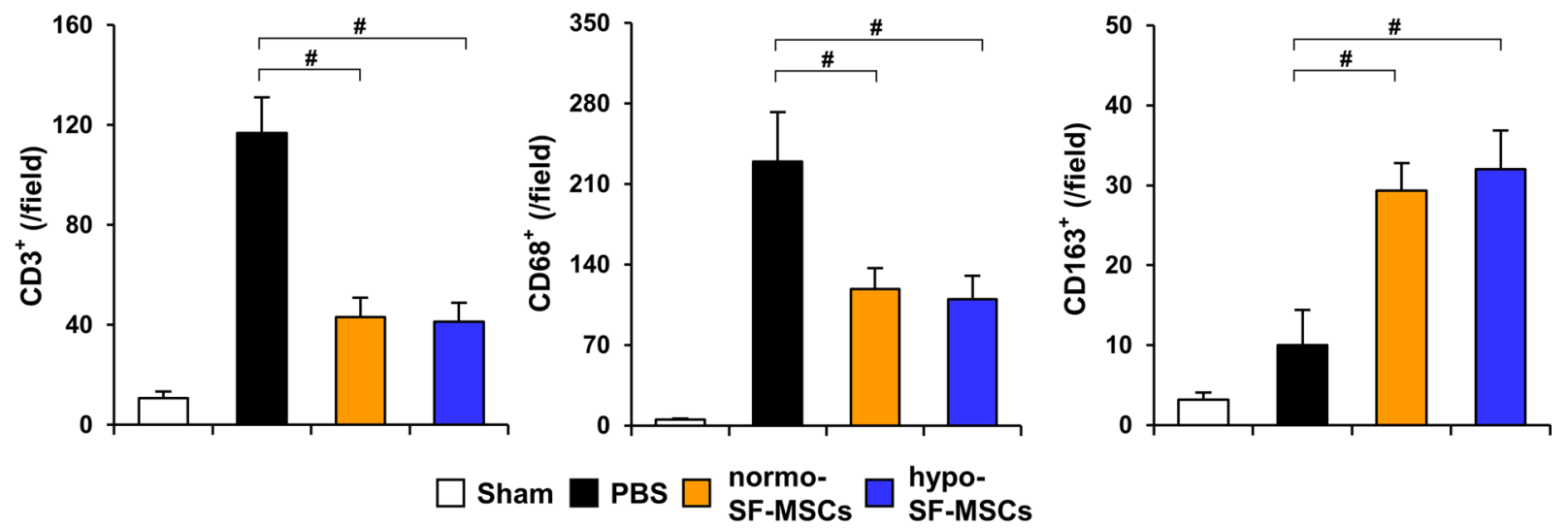

C
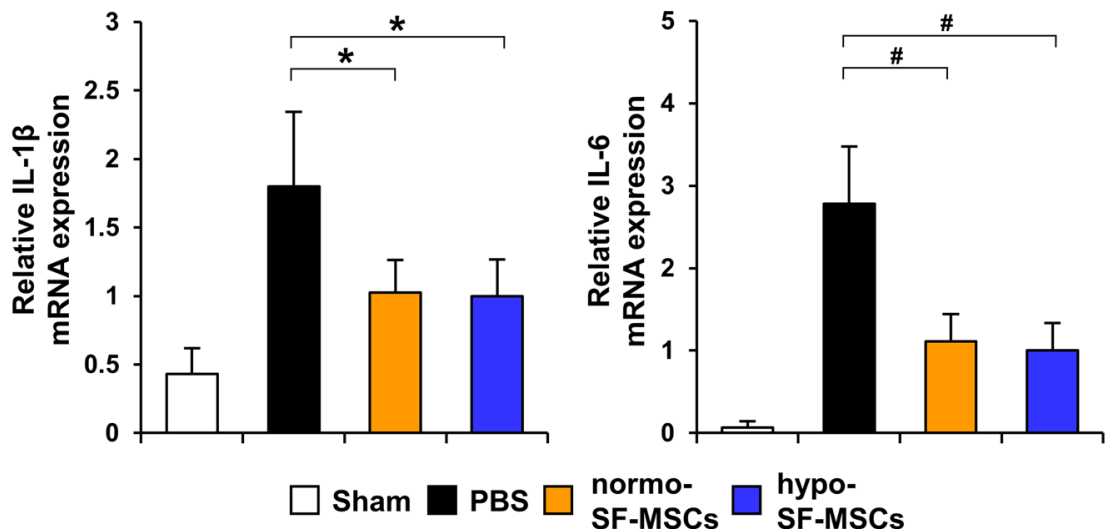

Fig. 3 Hypo-SF-MSCs suppress infiltration of inflammatory cells to the same extent as normo-SF-MSCs in IRI rats. a Representative images of immunohistochemical staining of CD3, CD68, and CD163 in kidney sections at day 7 post-IRI (scale bar =100 $\mu$ m). b Quantification of CD3-, CD68-, and CD163-positive cells ( $n=5$ in each group). $\mathbf{c}$ Expression levels of IL-1 $\beta$ and IL-6 mRNA in the kidney cortex of IRI rats at day 7 post-IRI ( $n=5$ in each group). Data are means \pm S.D. ${ }^{\#} P<0.01,{ }^{*} P<0.05$ (one-way ANOVA followed by Bonferroni's post-hoc test) 

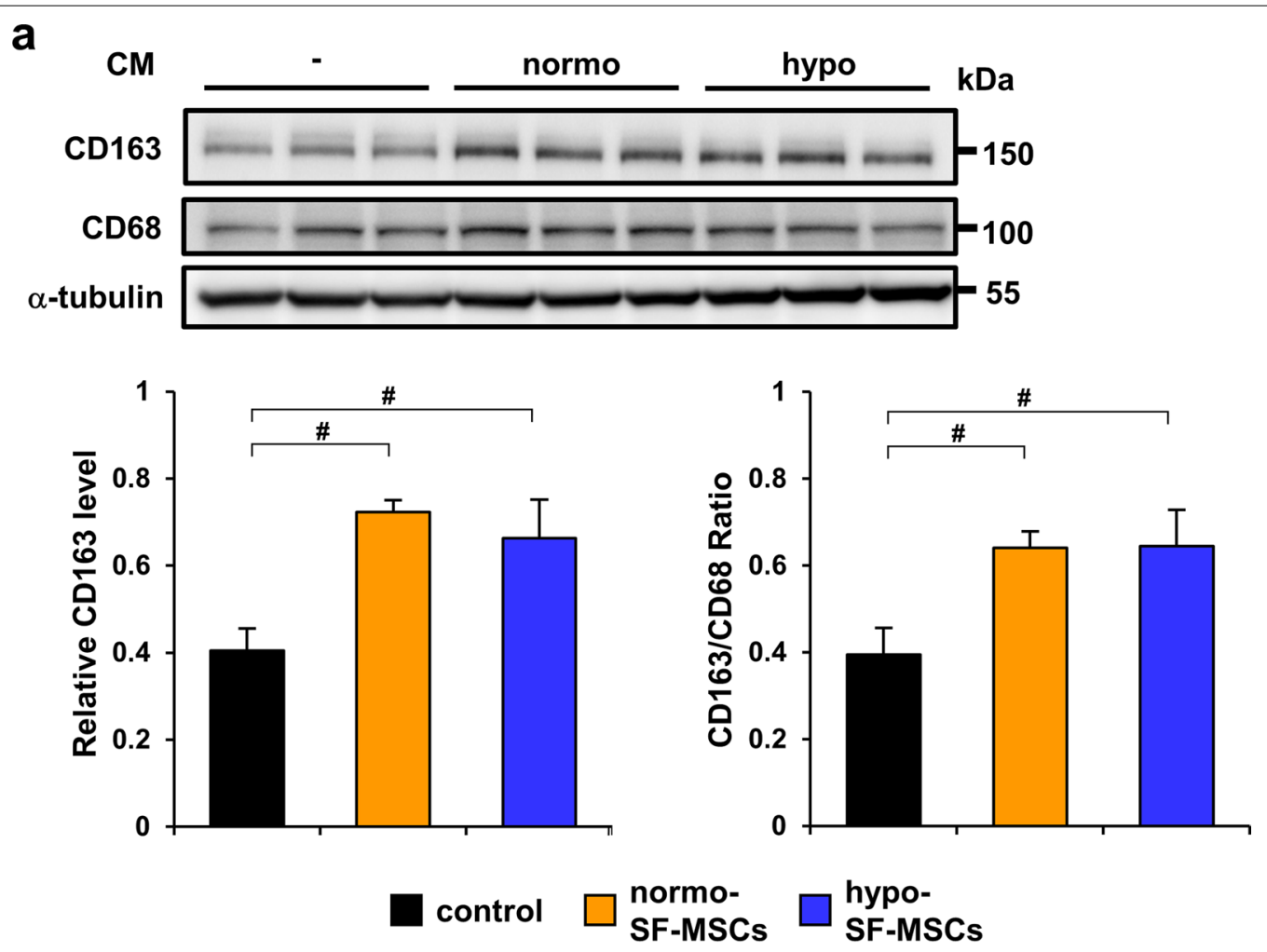

b

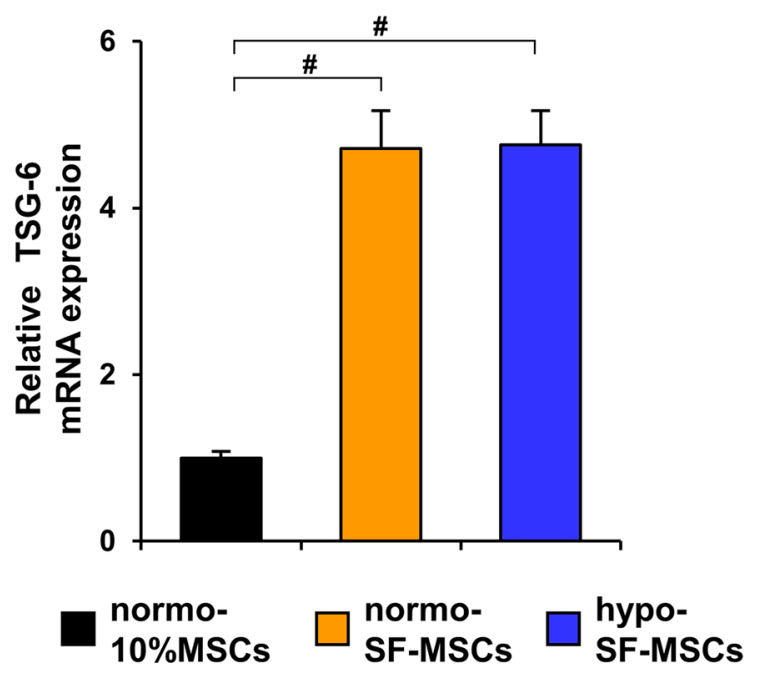

Fig. 4 Hypoxic preconditioning does not affect SF-MSC-induced changes in the macrophage phenotype from M1 to M2. a Western blot analysis of CD163 and CD68 in THP-1 macrophages incubated with conditioned medium from normo-SF-MSCs or hypo-SF-MSCs. Graph shows densitometric analysis of the CD163 expression level normalized to CD68 and GAPDH expression levels ( $n=5$ in each group). b Expression level of TSG-6 mRNA in normo-10\%MSCs, normo-SF-MSCs, and hypo-SF-MSCs ( $n=5$ in each group). Data are means \pm S.D. ${ }^{\#} P<0.01$ (one-way ANOVA followed by Bonferroni's post-hoc test)

siRNA (HGF siRNA/hypo-SF-MSC-CM) and negative control siRNA (NC siRNA/hypo-SF-MSC-CM). First, we confirmed successful knockdown of HGF in siRNAtransfected hypo-SF-MSCs by analyzing their conditioned medium (Fig. 6e). Moreover, to identify the effect of HGF secreted from MSCs on TGF- $\beta /$ Smad signaling, we examined the effect of NC siRNA/hypo-SF-MSC-CM and HGF siRNA/hypo-SF-MSC-CM on p-Smad2 and $\alpha$-SMA expression levels in TGF- $\beta 1$-stimulated HK-2 cells. As shown in Fig. 6f, TGF- $\beta 1$-induced expression of 


\section{a}
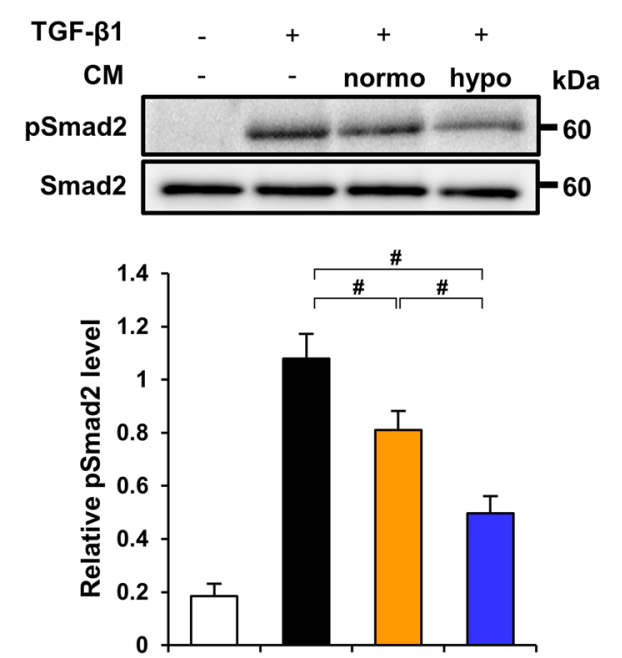

control b
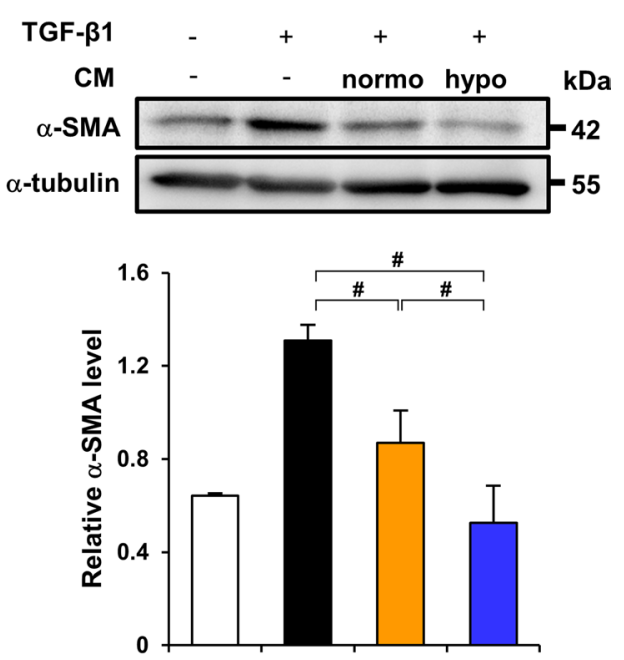

SF-MSC-CM

hypo-

Fig. 5 Conditioned medium from hypo-SF-MSCs strongly suppresses TGF- $\beta 1$-induced fibrotic changes in HK-2 cells. a Western blot analysis of p-Smad2 in HK-2 cells stimulated with TGF- $\beta 1$ for 30 min. Graph shows densitometric analysis of the p-Smad2 expression level normalized to the Smad 2 expression level ( $n=5$ in each group). $\mathbf{b}$ Western blot analysis of a-SMA in HK- 2 cells stimulated with TGF- $\beta 1$ for 24 h. Graph shows densitometric analysis of the a-SMA expression level normalized to the a-tubulin expression level ( $n=5$ in each group). Data are means \pm S.D. ${ }^{\#} P<0.01$ (one-way ANOVA followed by Bonferroni's post-hoc test)

p-Smad2 was suppressed by treatment with NC siRNA/ hypo-SF-MSC-CM. However, this inhibitory effect was weakened by treatment with HGF siRNA/hypo-SF-MSCCM (Fig. 6f). Similar results were observed for $\alpha$-SMA protein expression (Fig. 6g).

\section{Discussion}

We have shown that serum-free medium attenuates senescence of MSCs, promoting their proliferative capacity and that hypoxia preconditioning further enhances the proliferative capacity of SF-MSCs. Additionally, administration of hypo-SF-MSCs strongly ameliorated renal fibrosis in comparison with that of normo-SFMSCs in IRI rats, whereas the anti-inflammatory effect was almost equal for normo-SF-MSCs and hypo-SFMSCs. In vitro experiments revealed that hypo-SFMSC-CM more significantly inhibited TGF- $\beta /$ Smad signaling than normo-SF-MSC-CM. Moreover, we found that hypoxic preconditioning increased HGF secretion, whereas knockdown of HGF in hypo-SF-MSCs attenuated the inhibition of TGF- $\beta /$ Smad signaling. These findings suggest that serum-free conditions and hypoxic preconditioning synergistically enhance the anti-fibrotic effects of MSCs.

The persistence of inflammatory cell infiltration induces excessive production of extracellular matrix and fibrotic changes $[30,31]$. The TGF- $\beta /$ Smad signaling pathway is considered to play a central role in this process [32, 33]. In this study, although hypo-SF-MSCs and normo-SF-MSCs equivalently suppressed the infiltration of inflammatory cells and TGF- $\beta 1$ expression, hypo-SFMSCs strongly inhibited renal fibrosis compared with normo-SF-MSCs. These results are attributed to the fact that hypo-SF-MSCs more strongly suppressed TGF- $\beta /$

(See figure on next page.)

Fig. 6 Knockdown of HGF in hypo-SF-MSCs reduces the anti-fibrotic effect in TGF- $\beta 1$-stimulated HK-2 cells. The concentrations of vascular endothelial growth factor (VEGF) (a) and hepatocyte growth factor (HGF) (b) in each conditioned medium were measured by ELISAs ( $n=5$ in each group). c ELISA analysis showing the VEGF concentration in conditioned medium from SF-MSCs under hypoxia for various durations ( $n=5$ in each group). $\mathbf{d}$ ELISA analysis showing the VEGF concentration in conditioned medium from SF-MSCs cultured exposed to various $\mathrm{O}_{2}$ concentrations ( $n=5$ in each group). Hypo-SF-MSCs were transfected with HGF siRNA (HGF siRNA/hypo-SF-MSCs) or negative control siRNA (NC siRNA/ hypo-SF-MSCS). e The HGF concentration was measured in conditioned medium from NC siRNA/hypo-SF-MSCs and HGF siRNA/hypo-SF-MSCS using an ELISA ( $n=5$ in each group). f Western blot analysis of $p$-Smad 2 in HK-2 cells stimulated with TGF- $\beta 1$ for 30 min. Graph shows densitometric analysis of the $p$-Smad2 expression level normalized to the Smad2 expression level ( $n=5$ in each group). $\mathbf{g}$ Western blot analysis of a-SMA in HK-2 cells stimulated with TGF- $\beta 1$ for $24 \mathrm{~h}$. Graph shows densitometric analysis of the a-SMA expression level normalized to the a-tubulin expression level ( $n=5$ in each group). Data are means \pm S.D. ${ }^{\sharp} P<0.01,{ }^{*} P<0.05$ (one-way ANOVA followed by Bonferroni's post-hoc test or Student's t-test) 
a

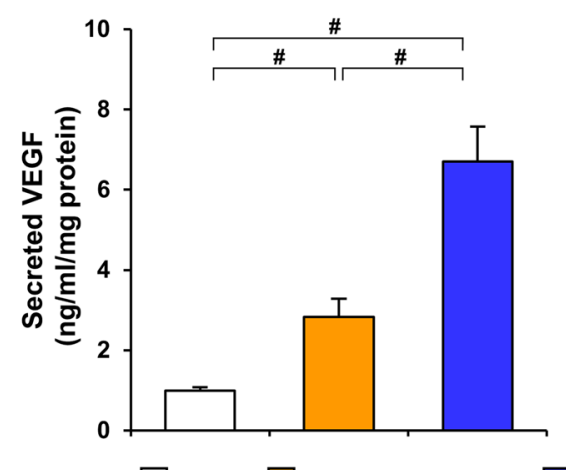

$\square$ HK-2 $\square$ normo-SF-MSCs $\square$ hypo-SF-MSCs

C

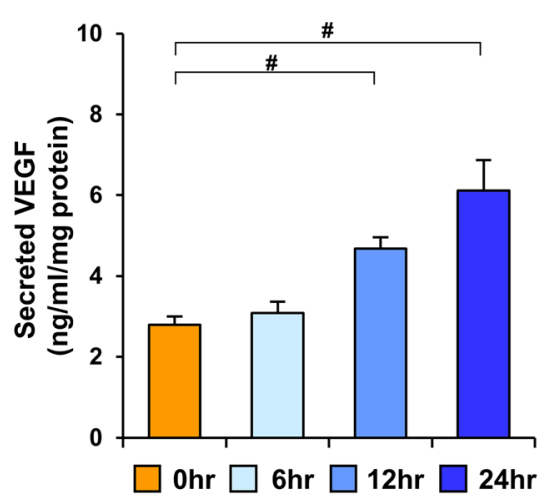

b

d
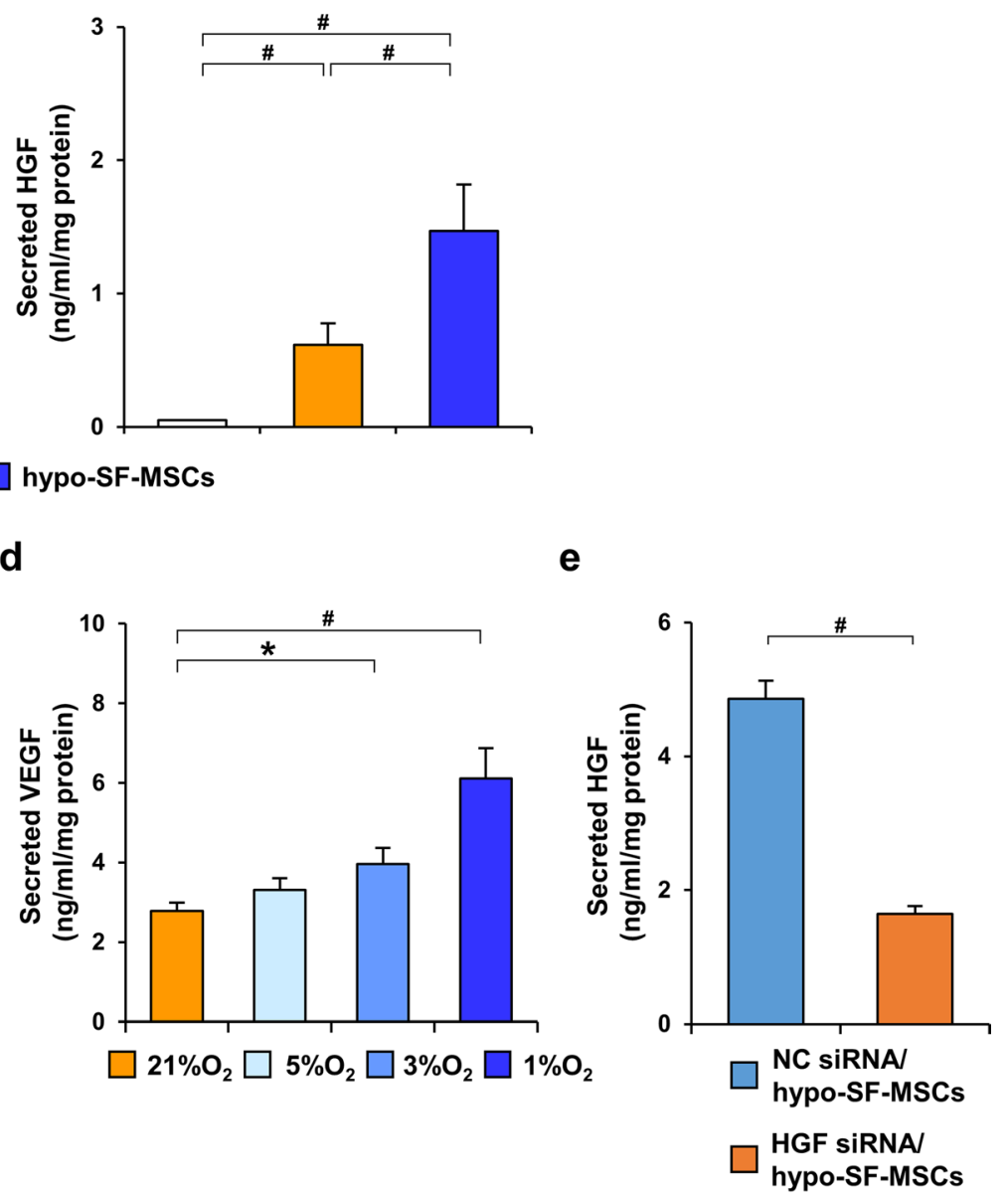

f
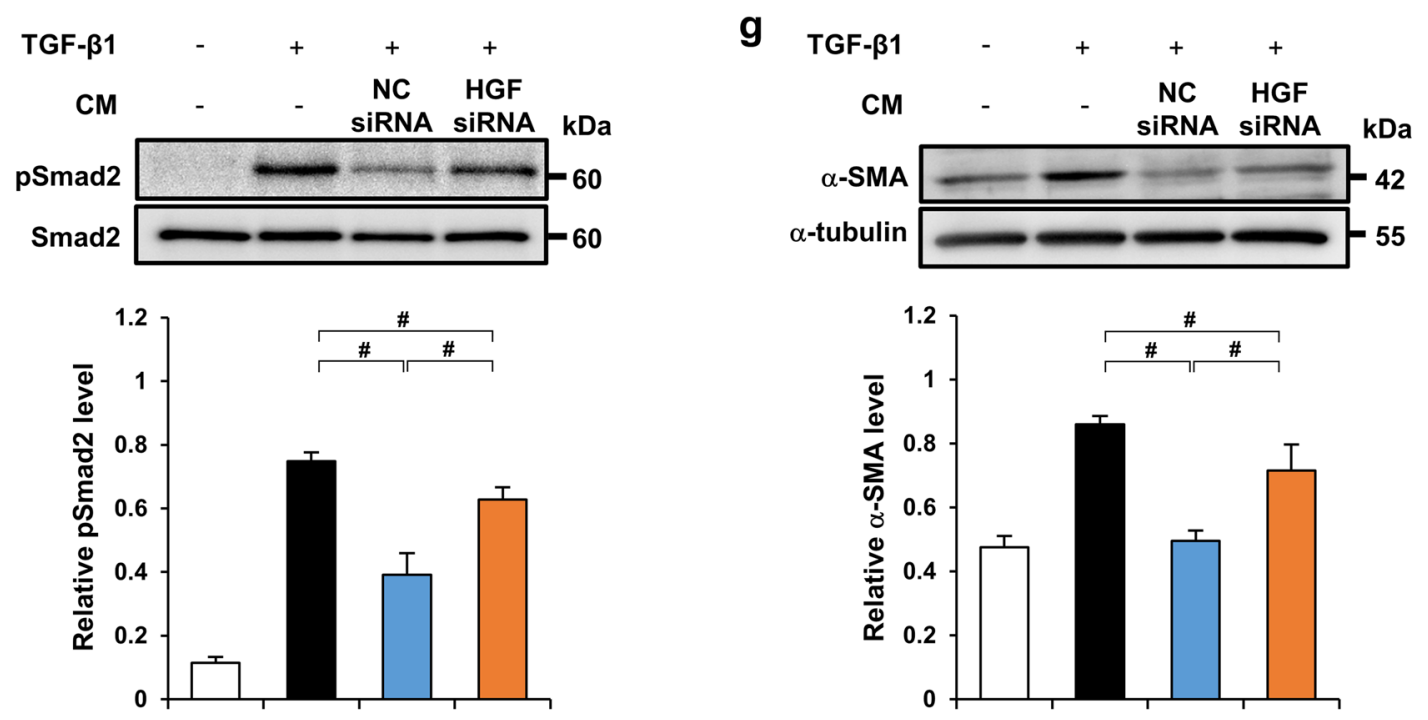

$\square$ control

DMEM

NC SiRNAI hypo-SF-MSC-CM

HGF SiRNA/ hypo-SF-MSC-CM

Fig. 6 (See legend on previous page.) 
Smad signaling, but not inflammation and TGF- $\beta 1$ itself, compared with normo-SF-MSCs. We have previously demonstrated that hypoxia-preconditioned 10\%MSCs enhance not only an immunosuppressive ability, but also a direct anti-fibrotic effect through inhibition of TGF- $\beta /$ Smad signaling [20]. Interestingly, this study indicated that hypoxic preconditioning only enhanced the direct anti-fibrotic effect of SF-MSCs, but not the antiinflammatory effect. This is probably because SF-MSCs had already exerted the strong anti-inflammatory effect through upregulation of TSG-6 as well as induction of polarization from M1 to M2 phenotype macrophages. Taken together, administration of hypo-SF-MSCs dramatically ameliorated renal fibrosis compared with that of normo-SF-MSCs by enhanced inhibition of the TGF- $\beta /$ Smad signaling pathway.

The therapeutic capacity of MSCs is considered to depend on their ability to secrete beneficial factors to repair the injured tissue $[34,35]$. We have previously reported that hypoxic preconditioning promotes VEGF and HGF secretion from 10\%MSCs [20]. In this study, hypoxic preconditioning enhanced secretion of these factors even under serum-free conditions. Moreover, TSG-6 expression was increased in MSCs under serum-free conditions and this increase was sustained even under hypoxic conditions. These results suggest that serum-free conditions and hypoxic preconditioning do not antagonize each other, but enhance paracrine activity. Additionally, we found that knockdown of HGF in hypo-SF-MSCs reduced the anti-fibrotic effect on TGF- $\beta 1$-induced fibrotic changes in HK-2 cells. Previous studies have shown that HGF antagonizes TGF- $\beta$ /Smad signaling [36, 37]. Therefore, our data suggest that hypoxic preconditioning promotes HGF secretion and thereby enhances inhibition of TGF- $\beta /$ Smad signaling, which leads to the pronounced anti-fibrotic effect of SF-MSCs.

For clinical application of MSCs, ex vivo culture is required to obtain a sufficient number of MSCs for treatments. Thus, the cell proliferative ability is important to obtain a large number of cells. In this study, we demonstrated that serum-free medium containing growth factors ameliorated cell senescence and promoted the cell proliferative ability and that hypoxic preconditioning further enhanced the proliferative ability of SF-MSCs. Therefore, also in terms of cell proliferative ability, preconditioning of SF-MSCs with hypoxia is a useful strategy. Sirt1 overexpression alleviates cellular senescence of MSCs and increases proliferative capacity [29]. However, genetic modification of MSCs requires complicated processes and cannot be performed easily. Therefore, hypoSF-MSCs are particularly suitable for ex vivo culture.

This study has a limitation, namely the absence of experimental data on renal functions. A previous study has shown that a decrease in renal fibrosis alone is not sufficient to improve renal functions [38]. Blood biochemistry is often assessed to evaluate renal functions after IRI. However, in our animal model, the unilateral renal pedicle was clamped without contralateral nephrectomy. Therefore, blood biochemistry could not be used to evaluate renal functions of the injured side after IRI.

\section{Conclusions}

In summary, administration of hypo-SF-MSCs further ameliorates renal fibrosis in IRI rats compared with normo-SF-MSCs with evidence that hypoxic preconditioning enhances the ability of SF-MSCs to inhibit the TGF- $\beta /$ Smad signaling pathway. We also revealed that the combination of serum-free conditions and hypoxic preconditioning enhances paracrine activity without competing with each other and that HGF plays a pivotal role in hypo-SF-MSC-mediated inhibition of TGF- $\beta /$ Smad signaling. Our results indicate that administration of hypoxia-preconditioned SF-MSCs has the potential to be a useful therapy to prevent the progression of renal fibrosis.

\begin{abstract}
Abbreviations
AKI: Acute kidney injury; CKD: Chronic kidney disease; DMEM: Dulbecco's modified Eagle's medium; ELISA: Enzyme-linked immunosorbent assay; FBS: Fetal bovine serum; HE: Hematoxylin and eosin; HGF: Hepatocyte growth factor; IL: Interleukin; IRI: Ischemia-reperfusion injury; MSCs: Mesenchymal stem cells; $10 \%$ MSCs: MSCs cultured in DMEM containing 10\% FBS; normo10\%MSCs: $10 \%$ MSCs cultured under $21 \%$ O2 conditions; SF-MSCs: MSCs cultured in serum-free medium; hypo-SF-MSCs: SF-MSCs cultured under 1\% O2 conditions; normo-SF-MSCs: SF-MSCs cultured under 21\% O2 conditions; hypo-SF-MSC-CM: Conditioned medium from hypo-SF-MSCs; normo-SFMSC-CM: Conditioned medium from normo-SF-MSCs; HGF siRNA/hypo-SFMSC-CM: Conditioned medium from hypo-SF-MSCs transfected with HGF siRNA; NC siRNA/hypo-SF-MSC-CM: Conditioned medium from hypo-SF-MSCS transfected with negative control siRNA; PBS group: IRI rats injected with PBS; hypo-SF-MSCs group: IRI rats injected with hypo-SF-MSCs; normo-SF-MSCs group: IRI rats injected with normo-SF-MSCs; p-Smad2: Phosphorylated Smad2; TSG-6: Tumor necrosis factor-a-induced protein 6; TGF: Transforming growth factor; VEGF: Vascular endothelial growth factor; WST: Water-soluble tetrazolium salts.
\end{abstract}

\section{Supplementary Information}

The online version contains supplementary material available at https://doi. org/10.1186/s13287-021-02548-7.

Additional file 1. Characterization of MSCs. a Flow cytometry showing expression of surface markers on normo-10\%MSCs. b, c Representative images of normo-10\%MSCs after staining with Oil Red $O$ and Alizarin Red $\mathrm{S}$ (scale bar $=100 \mu \mathrm{m})$.

Acknowledgements

We thank Ms. Miki Kagiya for technical assistance. We also thank Mitchell Arico from Edanz (https://jp.edanz.com/ac) for editing a draft of this manuscript. 


\section{Authors' contributions}

N.I. and A.N. designed the study; N.I., A.N., R.K., S.M., and S.T. carried out experiments; S.D., M.N., and T.M. analyzed the data; N.I. and A.N. drafted and revised the paper. All authors read and approved the final manuscript.

\section{Funding}

This study was supported in part by JSPS KAKENHI (Grant Number: JP20K08592) and the Japan Agency for Medical Research and Development (Research Project Number: 21 bk0104124h0001). A part of this work was carried out at the Analysis Center of Life Science, Natural Science Center for Basic Research and Development, Hiroshima University.

\section{Availability of data and materials}

The data that support the findings of this study are available from the corresponding author upon reasonable request.

\section{Declarations}

\section{Ethics approval and consent to participate}

The Medical Ethics Committee of Hiroshima Graduate School of Biomedical Science permitted collection of the bone marrow (Permit number: E-1089). All experimental procedures were approved by the Institutional Animal Care and Use Committee of Hiroshima University (Permit number: A16-83).

\section{Consent for publication}

Not applicable.

\section{Competing interests}

The Department of Stem Cell Biology and Medicine, Graduate School of Biomedical \& Health Sciences, Hiroshima University is a collaborative research laboratory funded by TWOCELLS Company, Limited. Dr. Maeda is the Vice manager of R\&D Division, Head Office of Research and Development of TWOCELLS Company, Limited. Except for the abovementioned disclosures, all other authors have declared that no conflict of interest exists.

\section{Author details}

${ }^{1}$ Department of Nephrology, Hiroshima University Hospital, 1-2-3 Kasumi, Minami-ku, Hiroshima, Hiroshima 734-8551, Japan. ${ }^{2}$ Center for Cause of Death Investigation Research, Graduate School of Biomedical \& Health Sciences, Hiroshima University, 1-2-3 Kasumi, Minami-ku, Hiroshima, Hiroshima 734-8553, Japan. ${ }^{3}$ Department of Forensic Medicine, Graduate School of Biomedical \& Health Sciences, Hiroshima University, 1-2-3 Kasumi, Minami-ku, Hiroshima, Hiroshima 734-8553, Japan. ${ }^{4}$ Department of Stem Cell Biology and Medicine, Graduate School of Biomedical \& Health Sciences, Hiroshima University, 1-2-3 Kasumi, Minami-ku, Hiroshima, Hiroshima 734-8553, Japan. ${ }^{5}$ TWOCELLS Company, Limited, 16-35 Hijiyama-honmachi, Minami-ku, Hiroshima 732-0816, Japan. ${ }^{6}$ Department of Surgery, Graduate School of Biomedical \& Health Sciences, Hiroshima University, 1-2-3 Kasumi, Minami-ku, Hiroshima, Hiroshima 734-8553, Japan.

Received: 7 May 2021 Accepted: 8 August 2021

Published online: 23 August 2021

\section{References}

1. Bouchard J, Acharya A, Cerda J, Maccariello ER, Madarasu RC, Tolwani AJ, et al. A prospective international multicenter study of AKI in the intensive care unit. Clin J Am Soc Nephrol. 2015;10:1324-31.

2. Parr SK, Matheny ME, Abdel-Kader K, Greevy RA Jr, Bian A, Fly J, et al. Acute kidney injury is a risk factor for subsequent proteinuria. Kidney Int. 2018:93:460-9.

3. van der Pol P, Schlagwein N, van Gijlswijk DJ, Berger SP, Roos A, Bajema $I M$, et al. Mannan-binding lectin mediates renal ischemia/reperfusion injury independent of complement activation. Am J Transplant. 2012:12:877-87.

4. Ronco C, Bellomo R, Kellum JA. Acute kidney injury. Lancet. 2019:394:1949-64.

5. Basile DP, Bonventre JV, Mehta R, Nangaku M, Unwin R, Rosner MH, et al. Progression after AKI: Understanding maladaptive repair processes to predict and identify therapeutic treatments. J Am Soc Nephrol. 2016;27:687-97.

6. Heung M, Steffick DE, Zivin K, Gillespie BW, Banerjee T, Hsu CY, et al. Acute kidney injury recovery pattern and subsequent risk of CKD: An analysis of Veterans Health Administration data. Am J Kidney Dis. 2016;67:742-52.

7. Venkatachalam MA, Weinberg LM, Kriz W, Bidani AK. Failed tubule recovery, $\mathrm{AKI}-\mathrm{CKD}$ transition, and kidney disease progression. J Am Soc Nephrol. 2015;26:1765-76.

8. Sato Y, Yanagita M. Immune cells and inflammation in AKI to CKD progression. Am J Physiol Renal Physiol. 2018;315:F1501-12.

9. Kern S, Eichler H, Stoeve J, Klüter H, Bieback K. Comparative analysis of mesenchymal stem cells from bone marrow, umbilical cord blood, or adipose tissue. Stem Cells. 2006;24:1294-301.

10. Keating A. Mesenchymal stromal cells: New directions. Cell Stem Cell. 2012;10:709-16.

11. Spees JL, Lee RH, Gregory CA. Mechanisms of mesenchymal stem/stromal cell function. Stem Cell Res Ther. 2016;7:125.

12. Phinney DG, Pittenger MF. Concise review: MSC-derived exosomes for cell-free therapy. Stem Cells. 2017;35:851-8.

13. Tögel F, Hu Z, Weiss K, Isaac J, Lange C, Westenfelder C. Administered mesenchymal stem cells protect against ischemic acute renal failure through differentiation-independent mechanisms. Am J Physiol Renal Physiol. 2005;289:F31-42

14. Qi S, Wu D. Bone marrow-derived mesenchymal stem cells protect against cisplatin-induced acute kidney injury in rats by inhibiting cell apoptosis. Int J Mol Med. 2013;32:1262-72.

15. Ren G, Zhang L, Zhao X, Xu G, Zhang Y, Roberts Al, et al. Mesenchyma stem cell-mediated immunosuppression occurs via concerted action of chemokines and nitric oxide. Cell Stem Cell. 2008;2:141-50.

16. Ren G, Su J, Zhang L, Zhao X, Ling W, L'huillie A, et al. Species variation in the mechanisms of mesenchymal stem cell-mediated immunosuppression. Stem Cells. 2009;27:1954-62.

17. Li M, Zeng L, Liu S, Dangelmajer S, Kahlert UD, Huang H, et al. Transforming growth factor- $\beta$ promotes homing and therapeutic efficacy of human mesenchymal stem cells to glioblastoma. J Neuropathol Exp Neurol. 2019:78:315-25.

18. Kanai R, Nakashima A, Doi S, Kimura T, Yoshida K, Maeda S, et al. Interferon- $\gamma$ enhances the therapeutic effect of mesenchymal stem cells on experimental renal fibrosis. Sci Rep. 2021;11:850.

19. Yu J, Yin S, Zhang W, Gao F, Liu Y, Chen Z, et al. Hypoxia preconditioned bone marrow mesenchymal stem cells promote liver regeneration in a rat massive hepatectomy model. Stem Cell Res Ther. 2013:4:83.

20. Ishiuchi N, Nakashima A, Doi S, Yoshida K, Maeda S, Kanai R, et al. Hypoxia-preconditioned mesenchymal stem cells prevent renal fibrosis and inflammation in ischemia-reperfusion rats. Stem Cell Res Ther. 2020;11:130.

21. Numasawa Y, Kimura T, Miyoshi S, Nishiyama N, Hida N, Tsuji H, et al. Treatment of human mesenchymal stem cells with angiotensin receptor blocker improved efficiency of cardiomyogenic transdifferentiation and improved cardiac function via angiogenesis. Stem Cells. 2011;29:1405-14.

22. Pourjafar M, Saidijam M, Mansouri K, Ghasemibasir H, Karimi Dermani F, Najafi R. All-trans retinoic acid preconditioning enhances proliferation, angiogenesis and migration of mesenchymal stem cell in vitro and enhances wound repair in vivo. Cell Prolif. 2017;50:e12315.

23. Liu X, Chen H, Zhu W, Chen H, Hu X, Jiang Z, et al. Transplantation of SIRT1-engineered aged mesenchymal stem cells improves cardiac function in a rat myocardial infarction model. J Heart Lung Transplant. 2014;33:1083-92.

24. Liu F, Xie J, Zhang X, Wu Z, Zhang S, Xue M, et al. Overexpressing TGF- $\beta 1$ in mesenchymal stem cells attenuates organ dysfunction during CLPinduced septic mice by reducing macrophage-driven inflammation. Stem Cell Res Ther. 2020:11:378.

25. Yoshida K, Nakashima A, Doi S, Ueno T, Okubo T, Kawano KI, et al. Serumfree medium enhances the immunosuppressive and antifibrotic abilities of mesenchymal stem cells utilized in experimental renal fibrosis. Stem Cells Transl Med. 2018;7:893-905.

26. Wagner W, Ho AD, Zenke M. Different facets of aging in human mesenchymal stem cells. Tissue Eng Part B Rev. 2010;16:445-53.

27. Pi C, Yang $Y$, Sun $Y$, Wang $H$, Sun $H$, Ma M, et al. Nicotinamide phosphoribosyltransferase postpones rat bone marrow mesenchymal stem cell 
senescence by mediating $\mathrm{NAD}^{+}$-Sirt1 signaling. Aging (Albany NY) 2019;11:3505-22.

28. Yuan $H F$, Zhai C, Yan XL, Zhao DD, Wang JX, Zeng $Q$, et al. SIRT1 is required for long-term growth of human mesenchymal stem cells. J Mol Med. 2012;90:389-400.

29. Chen H, Liu X, Zhu W, Chen H, Hu X, Jiang Z, et al. SIRT1 ameliorates agerelated senescence of mesenchymal stem cells via modulating telomere shelterin. Front Aging Neurosci. 2014;6:103.

30. Meng XM, Nikolic-Paterson DJ, Lan HY. Inflammatory processes in renal fibrosis. Nat Rev Nephrol. 2014;10:493-503.

31. Djudjaj S, Martin IV, Buhl EM, Nothofer NJ, Leng L, Piecychna M, et al. Macrophage migration inhibitory factor limits renal inflammation and fibrosis by counteracting tubular cell cycle arrest. J Am Soc Nephrol. 2017;28:3590-604.

32. Meng XM, Nikolic-Paterson DJ, Lan HY. TGF- $\beta$ : The master regulator of fibrosis. Nat Rev Nephrol. 2016;12:325-38.

33. Szeto SG, Narimatsu M, Lu M, He X, Sidiqi AM, Tolosa MF, et al. YAP/TAZ are mechanoregulators of TGF- $\beta$-Smad Signaling and renal fibrogenesis. J Am Soc Nephrol. 2016;27:3117-28.

34. Mirotsou M, Jayawardena TM, Schmeckpeper J, Gnecchi M, Dzau VJ. Paracrine mechanisms of stem cell reparative and regenerative actions in the heart. J Mol Cell Cardiol. 2011;50:280-9.
35. Pawitan JA, Bui TA, MubarokW, Antarianto RD, Nurhayati RW, Dilogo $\mathbb{H}_{\text {, }}$ et al. Enhancement of the therapeutic capacity of mesenchymal stem cells by genetic modification: A systematic review. Front Cell Dev Biol. 2020;8:587776.

36. Dai C, Liu Y. Hepatocyte growth factor antagonizes the profibrotic action of TGF-beta1 in mesangial cells by stabilizing Smad transcriptional corepressor TGIF. J Am Soc Nephrol. 2004;15:1402-12.

37. Liu Y. Hepatocyte growth factor in kidney fibrosis: Therapeutic potential and mechanisms of action. Am J Physiol Renal Physiol. 2004:287:F7-16.

38. Allinovi M, De Chiara L, Angelotti ML, Becherucci F, Romagnani P. Anti-fibrotic treatments: A review of clinical evidence. Matrix Biol. 2018;68-69:333-54.

\section{Publisher's Note}

Springer Nature remains neutral with regard to jurisdictional claims in published maps and institutional affiliations.
Ready to submit your research? Choose BMC and benefit from:

- fast, convenient online submission

- thorough peer review by experienced researchers in your field

- rapid publication on acceptance

- support for research data, including large and complex data types

- gold Open Access which fosters wider collaboration and increased citations

- maximum visibility for your research: over $100 \mathrm{M}$ website views per year

At BMC, research is always in progress.

Learn more biomedcentral.com/submissions 